\title{
EVALUACIÓN DE PARÁMETROS DE CALIDAD FíSICO- QUÍMICA, MICROBIOLÓGICA Y SENSORIAL EN TOMATE DESHIDRATADO COMERCIAL (Lycopersicum esculentum)
}

\section{QUALITY EVALUATION OF PHYSICO-CHEMICAL, MICROBIOLOGICAL AND SENSORIAL PARAMETERS OF DRIED TOMATO (Lycopersicum esculentum)}

\begin{abstract}
Diana Catalina Moreno ${ }^{1}$, Hernán Mauricio Sierra ${ }^{2}$, Consuelo Díaz-Moreno ${ }^{3}$
${ }^{1}$ I.Q. Instituto de Ciencia y Tecnología de Alimentos-ICTA, Universidad Nacional de Colombia, Carrera 30 No. 45-03, BogotáColombia, e-mail: dcmorenog@unal.edu.co; ${ }^{2}$ I.Q. Instituto de Ciencia y Tecnología de Alimentos-ICTA, Universidad Nacional de Colombia, Carrera 30 No. 45-03, Bogotá-Colombia, e-mail: hmsierrah@bt.unal.edu.co; ${ }^{3}$ I.Ali. Ph.D. Instituto de Ciencia y Tecnología de Alimentos-ICTA, Universidad Nacional de Colombia, Carrera 30 No. 45-03, Bogotá-Colombia, e-mail: amcdiazmo@unal.edu.co
\end{abstract}

Rev. U.D.C.A Act. \& Div. Cient. 17(1): 131 - 138, Enero-Junio, 2014

\section{RESUMEN}

El tomate es una de las hortalizas más importantes en el mundo, por su nivel de producción y de consumo. Debido a su naturaleza perecedera tiene un difícil manejo postcosecha, lo que ocasiona pérdidas importantes de producto durante su almacenamiento y comercialización, razón importante para considerar métodos de conservación, como la deshidratación y así extender su vida útil. Este trabajo tuvo como objetivo establecer los parámetros fisicoquímicos, microbiológicos y sensoriales, que influyen en la calidad de muestras comerciales de tomate deshidratado. Para el estudio, se utilizaron muestras del mercado local y se analizaron los parámetros humedad, actividad de agua y $\mathrm{pH}$, mediante las técnicas estandarizadas de la AOAC; color, utilizando el sistema CIE, con coordenadas L*a*b* y análisis de textura, aplicando la metodología del TPA (Texture Profile Analysis). Adicionalmente, se verificó la calidad microbiológica del producto, según la metodología sugerida por el INVIMA; finalmente, se hizo una prueba sensorial hedónica dirigida a consumidores, para correlacionar con los análisis instrumentales. Se obtuvo que el $\mathrm{pH}$ no tiene influencia en el tomate deshidratado comercial y que el color y la textura, además de presentar diferencias significativas entre muestras $(p<0.05)$, son los parámetros más importantes en cuanto a la calidad sensorial del tomate, ya que el consumidor busca una color igual al tomate fresco y como producto deshidratado prefiere un producto blando y con fracturabilidad alta.

\section{SUMMARY}

Tomato is one of the world's most important vegetables because of its high production level and consumption. Due to its perishable nature, post-harvest handling is difficult, which causes major losses during storage and marketing, important reason to consider conservation methods such as dehydration, in order to extend its shelf life. This work aimed to establish the physicochemical, microbiological and sensory quality parameters of commercial samples of dried tomato. For the study samples of the local market were used and humidity, water activity and $\mathrm{pH}$ using standardized techniques AOAC coordinates CIE $L * a * b *$ for color and TPA (Texture Profile analysis) methodology for texture analysis were established. Further the microbiological quality of the product according to the methodology used by INVIMA was verified. Finally a hedonic sensory test directed to consumers to be correlated with instrumental analysis was carried out. Results showed that $\mathrm{pH}$ has no influence on the commercially dehydrated tomatoes. Color and texture, besides presenting significant differences between samples $(p<0.05)$ were the most important parameters related to the sensorial quality of tomato; consumers prefer a color with similar characteristics as that of fresh tomato and also prefer a soft product with high fracturability.

Key words: Color, texture, quality, $\mathrm{pH}$.

Palabras clave: Color, textura, calidad, $\mathrm{pH}$. 


\section{INTRODUCCIÓN}

Debido al alto contenido de agua que presenta el tomate, aproximadamente el 94\%, surge la necesidad de buscar alternativas tecnológicas para conservar el producto. Uno de los métodos de conservación que se puede aplicar es la deshidratación, que disminuye el agua libre disponible en el producto a actividades de agua inferiores 0,4 en donde las reacciones microbiológicas y enzimáticas se ven disminuidas (Ashebir et al. 2009; Monsalve \& Machado, 2007).

La deshidratación es una de las tecnologías más frecuentes en la agroindustria, por ser la operación unitaria, en la cual, se elimina la humedad de un material por la acción de determinadas condiciones ambientales de temperatura y de humedad relativa, con el objetivo de reducir la actividad microbiana y reacciones de deterioro del producto (Akdeniz et al. 2012; Chang et al. 2006; Heredia et al. 2007; Ratti, 2001).

El tomate deshidratado, se caracteriza mediante algunas propiedades físico-químicas, como el $\mathrm{pH}$, sólidos solubles, humedad, actividad de agua (Khazaei et al. 2008) y algunos parámetros microbiológicos, como mesófilos, coliformes, hongos y levaduras (Hossain et al. 2008). El contenido de humedad del tomate deshidratado es reducido a $15 \%$ (Brooks et al. 2008; Zanoni et al. 1998).

Estudios indican que la deshidratación de las rodajas de tomate provoca un aumento de los sólidos solubles y de la acidez, mientras que el $\mathrm{pH}$ y el ácido ascórbico disminuyen (Khazaei et al. 2008; Polenta et al. 2006; Thybo et al. 2006). Algunos de los parámetros que se han medido con frecuencia en productos deshidratados son el color y la textura, como parámetros de calidad sensorial del producto final (Heredia et al. 2007). El cambio de color en los alimentos durante el proceso térmico es causado por reacciones de degradación de pigmentos, especialmente carotenoides y clorofila, reacciones de pardeamiento, como la condensación de Maillard de hexosas y amino componentes, junto con la oxidación del ácido ascórbico (Ashebir et al. 2009; Barreiro et al. 1997; Lozano \& Ibarz, 1997); por lo tanto, los valores finales de los parámetros de color pueden ser usados como indicadores de calidad para evaluar el deterioro, debido al procesamiento térmico (Shi et al. 1999; Shin \& Bhowmik, 1995).

Un producto alimenticio debe seguir estrictas normas microbiológicas para no convertirse en un riesgo para la salud humana. El gobierno de la República de Colombia, mediante el Ministerio de Protección Social, está en proceso de trámite del Proyecto de Resolución, mediante el cual, se instaura el reglamento técnico sobre los requisitos sanitarios que deben cumplir las frutas que se procesen, empaquen, transporten, importen y comercialicen en el territorio nacional y se disponen los requisitos microbiológicos de frutas deshidratadas o desecadas, estableciendo como parámetros un recuento de mohos y levaduras menor a 100UFC/g y ausencia de Salmonella, por cada 25g (Ministerio de la Protección Social de Colombia, 2011).

Actualmente, en Colombia no existe reglamentación que establezca criterios de calidad microbiológicos para gran parte del sector alimentario; debido a esto, los productores de fruta deshidratada no tienen normas microbiológicas a cumplir, lo que dificulta las condiciones de estandarización del proceso de deshidratación. Este trabajo utiliza como referencia normas internacionales y guías técnicas para frutas y vegetales deshidratados. La norma británica considera los parámetros microbiológicos y establece un número máximo de coliformes totales de $25 \mathrm{UFC} / \mathrm{g}$, ausencia de Salmonella y menos de $10^{5} \mathrm{UFC} / \mathrm{g}$, mesófilos aerobios (Gilbert et al. 2000). La norma peruana muestra las características y establece un número máximo de mesófilos aerobios de 100 $\mathrm{UFC} / \mathrm{g}$, coliformes totales menores a $500 \mathrm{UFC} / \mathrm{g}$, hongos y levaduras menores a $100 \mathrm{UFC/g}$ y ausencia de Salmonella (Ministerio de Salud del Perú, 2003). Finalmente, se revisó la norma brasilera, que ordena como criterio $200 \mathrm{UFC} / \mathrm{g}$, de coliformes totales y ausencia de Salmonella, en $25 \mathrm{~g}$ (Ministério da Saúde Brasil, 1978).

El estudio tuvo como objetivo evaluar las propiedades fisicoquímicas, microbiológicas y sensoriales de muestras comerciales de tomate deshidaratado.

\section{MATERIALES Y MÉTODOS}

Se analizaron siete muestras comerciales de producción nacional de tomate deshidratado con diferentes marcas, adquiridas en el mercado local de la ciudad de Bogotá. Estas muestras, se tomaron de acuerdo a su frecuencia en las grandes superficies, almacenadas en condiciones de temperatura $19^{\circ} \mathrm{C}$ y un contenido de humedad del $75 \%$.

La humedad, se midió utilizando la metodología de sólidos totales en frutas y en productos de frutas de la AOAC 920.151. Se secó la muestra a $105^{\circ} \mathrm{C}$, durante dos horas, controlando el peso antes y después del secado (AOAC. 920.151, 2002); se realizaron tres réplicas experimentales.

La determinación de $\mathrm{pH}$, se realizó de acuerdo con la Norma Técnica Colombiana 4592, se tomó una parte de la muestra en pequeños trozos y se eliminaron las semillas. Luego, se pusieron los trozos de muestra en un vaso de precipitado; se agregó dos o tres veces su masa de agua destilada y se calienta en un baño de agua, durante 30 minutos; se macera y se lee el $\mathrm{pH}$ directamente sobre la escala del aparato, con precisión de 0,05 unidades de $\mathrm{pH}$, hasta que se obtenga un valor constante; las mediciones se hicieron a temperatura ambiente (ICONTEC. NTC 4592, 1999). 
La actividad de agua, se midió de manera instrumental directa, en un activímetro GBX Modelo FAst-Lab; la medición, se basa en la saturación del aire dentro de la cámara de muestreo del equipo con la humedad presente en el producto, alcanzándose el punto de rocío, que es cuantificado mediante la capacidad reflectiva del espejo de condensación (GBX, 2008). Las determinaciones, se realizaron por triplicado, para cada muestra evaluada.

El color, se midió de manera instrumental colorímetro Minolta Modelo C-300, utilizando el sistema CIE y las coordenadas L*a*b*, para la descripción del color; se hicieron tres réplicas a cada muestra. Posteriormente, se calculó el ángulo de tono (hab), que se utiliza para establecer el tono del alimento: rojo, amarillo, verde o azul, o una combinación de dos de ellos consecutivos (Batu, 2004).

El croma $\left(C^{*}{ }_{a b}\right)$, que es medida de la pureza del color; los colores no saturados contienen una proporción de luz blanca. Estos parámetros, se hallan mediante las Ecuaciones (1) y (2) (Heredia et al. 2007).

$$
\begin{gathered}
h_{a b}=\tan ^{-1}\left(b^{*} / a^{*}\right)(1) \\
C_{a b}^{*}=\sqrt{\left(a^{2}+b^{2}\right)}(2)
\end{gathered}
$$

Para la medida de parámetros texturales, se utilizó un texturómetro SMS Modelo TA-XTplus. La caracterización de las muestras, se realizó mediante una prueba de Análisis de Perfil de Textura (TPA) (Kek et al. 2013), empleando el disco de compresión de $75 \mathrm{~mm}$; la distancia de ingreso a la muestra para la prueba fue de $5 \mathrm{~mm}$. Debido a la dispersión que pueden presentar las propiedades texturales, se hicieron cinco réplicas para cada muestra evaluada. Se evaluaron los parámetros de dureza, de fracturabilidad, de cohesividad, de elasticidad y de masticabilidad.
Se realizaron pruebas de calidad microbiológica, para identificar mesófilos, mohos y levaduras, coliformes totales y Salmonella sp. Todos los métodos de identificación y recuento utilizados son los normalizados por el Instituto Nacional para la Vigilancia de Medicamentos y Alimentos (INVIMA, 1998).

Se adaptó una prueba sensorial de aceptación de escala numérica, aplicada a 33 panelistas, para poder establecer diferencias significativas, con $5 \%$ de significancia, en la cual, cada panelista codificaba las muestras de manera ordinal, de acuerdo a su preferencia (Sidel \& Moskowitz, 1971; Sidel \& Stone, 1976).

El análisis estadístico de los datos, se realizó mediante un análisis de varianza (ANOVA), para verificar la influencia de cada parámetro en las muestras evaluadas; adicionalmente, se aplicó una prueba de Tukey, con el propósito de observar si existen diferencias significativas entre los parámetros de calidad evaluados (Montgomery, 2002). A las propiedades texturales, se les hizo Análisis de Componentes Principales (PCA), para observar efectos simultáneos entre las variables.

\section{RESULTADOS Y DISCUSIÓN}

La humedad, la actividad de agua y el $\mathrm{pH}$ de las muestras estudiadas, se presentan en la tabla 1 . Se observaron diferencias estadísticas en el valor de la humedad de las muestras 2, 4, 5 y 6; esta variación, se debe a las diferencias que presentan cada una de las muestras comerciales en su almacenamiento inicial, ya que al ser muestras de diferentes marcas vienen almacenadas en distintos materiales.

La muestra 7 presentó diferencias en la variable humedad, con respecto a las demás $(p<0,05)$. La actividad de agua varia en un rango de 0,525 y 0,771 , valores que no son característicos para un alimento deshidratado (Ratti, 2009),

Tabla 1 . Humedad, actividad de agua y $\mathrm{pH}$ en muestras comerciales de tomate deshidratado, $(\mathrm{p}>0.05)$.

\begin{tabular}{|c|c|c|c|}
\hline Muestra & $\begin{array}{c}\text { Humedad } \\
\text { (kg agua/kg de muestra) }\end{array}$ & $\begin{array}{c}\text { Actividad de Agua } \\
\text { aw }\end{array}$ & pH \\
\hline 1 & $0,224 \pm 0,006 \mathrm{~b}$ & $0,636 \pm 0,006 \mathrm{c}$ & $3,62 \pm 0,02 \mathrm{a}$ \\
\hline 2 & $0,163 \pm 0,001 \mathrm{a}$ & $0,567 \pm 0,001 \mathrm{~b}$ & $4,09 \pm 0,02 \mathrm{ab}$ \\
\hline 3 & $0,280 \pm 0,015 \mathrm{c}$ & $0,721 \pm 0,008 \mathrm{~d}$ & $4,15 \pm 0,02 \mathrm{ab}$ \\
\hline 4 & $0,169 \pm 0,003 \mathrm{a}$ & $0,525 \pm 0,013 \mathrm{a}$ & $4,26 \pm 0,01 \mathrm{~b}$ \\
\hline 5 & $0,173 \pm 0,010 \mathrm{a}$ & $0,603 \pm 0,006 \mathrm{bc}$ & $3,57 \pm 0,02 \mathrm{a}$ \\
\hline 6 & $0,159 \pm 0,009 \mathrm{a}$ & $0,530 \pm 0,006 \mathrm{a}$ & $4,34 \pm 0,01 \mathrm{~b}$ \\
\hline 7 & $0,388 \pm 0,022 \mathrm{~d}$ & $0,771 \pm 0,005 \mathrm{e}$ & $4,11 \pm 0,01 \mathrm{ab}$ \\
\hline
\end{tabular}

Media \pm SD (Desviación estándar). Promedios con letras diferentes en una misma columna presentan diferencias significativas (Tukey, $\mathrm{p}>0.05$ ). 
dejando agua libre disponible para reacciones de deterioro microbiano.

En la medida de $\mathrm{pH}$, la dispersión de las muestras no es significativa y solamente se pueden distinguir dos grupos de muestras; adicionalmente, no se encuentra ninguna correlación del pH con respecto a la humedad del producto, puesto que su correlación de Pearson es 0,1 , indicando una relación entre las variables positiva, pero no significativa; sin embargo, varios estudios afirman que al descender la humedad el pH también disminuye (Ashebir et al. 2009; Khazaei et al. 2008).

La tabla 2 muestra las coordenadas de color obtenidas para las diferentes muestras estudiadas. El parámetro de luminosidad $\left(L^{*}\right)$ no presenta diferencias significativas para ninguna muestra, por lo cual, se infiere que el parámetro de luminosidad no influye en muestras de tomate deshidratado comercial.

En el análisis de los parámetros de color, de tono y de cromaticidad, las muestras se encuentran en el punto intermedio del primer cuadrante $\left(0\right.$ a $\left.90^{\circ}\right)$, tomando una tonalidad naranja; la cromaticidad $\left(\mathrm{C}^{*}{ }_{a b}\right)$ indica la saturación del co- lor; Olorunda et al. (1990) establecieron una escala de este parámetro en tomate: si es mayor a 20 unidades, el color del producto deshidratado cumple los parámetros de buena aceptación sensorial; para todos los casos, se cumplió con esta escala, indicando que todas las muestras son aceptadas; sensorialmente tienen valores estadísticamente iguales, excepto la muestra número 6 , que presenta una saturación más baja que las otras muestras, derivado de la reacción de caramelización de azúcares, ocasionada por el tratamiento térmico y causa importante de la degradación de color, alterando, principalmente, el valor de la cromaticidad, afectando no sólo el color sino que, también, produciendo cambios en las características bioactivas (Shi et al. 1999).

Al correlacionar el tono y la cromaticidad, la muestra de color más aceptado para comercialización sería aquella de un ángulo de tono más bajo y un croma más elevado; la muestra 1 cumple con estas condiciones.

Como se observa en la figura 1 (Score Plot), que se refiere a la influencia de las muestras y, la figura 2 (Loading Plot), que se refiere la influencia de las variables; las gráficas del Análisis de Componentes Principales muestran cómo los dos

Tabla 2. Coordenadas de color, cromaticidad y tono en muestras de tomate deshidratado comercial ( $>>0.05)$.

\begin{tabular}{|c|l|l|l|l|l|}
\hline Muestra & \multicolumn{1}{|c|}{$\mathrm{L}^{*}$} & \multicolumn{1}{c|}{$\mathrm{a}^{*}$} & \multicolumn{1}{c|}{$\mathrm{b}^{*}$} & \multicolumn{1}{c|}{$\mathrm{C}_{\mathrm{ab}}$} & \multicolumn{1}{c|}{ hab } \\
\hline 1 & $36,3 \pm 2,9 \mathrm{a}$ & $28,2 \pm 3,2 \mathrm{a}$ & $21,0 \pm 4,9 \mathrm{a}$ & $35,3 \pm 4,8 \mathrm{~b}$ & $36,0 \pm 5,72 \mathrm{a}$ \\
\hline 2 & $41,0 \pm 7,1 \mathrm{a}$ & $23,5 \pm 4,7 \mathrm{ab}$ & $32,5 \pm 4,8 \mathrm{~b}$ & $40,3 \pm 5,7 \mathrm{~b}$ & $54.4 \pm 5,15 \mathrm{~b}$ \\
\hline 3 & $32,0 \pm 2,5 \mathrm{a}$ & $23,2 \pm 5,2 \mathrm{ab}$ & $18,7 \pm 2,2 \mathrm{a}$ & $30,0 \pm 4,5 \mathrm{ab}$ & $39,5 \pm 6.87 \mathrm{a}$ \\
\hline 4 & $41,5 \pm 3,5 \mathrm{a}$ & $22,2 \pm 3,9 \mathrm{~b}$ & $27,1 \pm 3,9 \mathrm{c}$ & $35,2 \pm 4,2 \mathrm{~b}$ & $50,4 \pm 5,72 \mathrm{~b}$ \\
\hline 5 & $36,7 \pm 6,4 \mathrm{a}$ & $27,4 \pm 3,3 \mathrm{a}$ & $22,2 \pm 9,1 \mathrm{ab}$ & $35,6 \pm 8,3 \mathrm{~b}$ & $37,8 \pm 7,44 \mathrm{a}$ \\
\hline 6 & $31,7 \pm 7,1 \mathrm{a}$ & $15,4 \pm 6,2 \mathrm{c}$ & $20,1 \pm 7,0 \mathrm{a}$ & $25,7 \pm 8,0 \mathrm{a}$ & $53,2 \pm 12,0 \mathrm{~b}$ \\
\hline 7 & $33,3 \pm 3,5 \mathrm{a}$ & $22,1 \pm 4,4 \mathrm{~b}$ & $21,7 \pm 3,0 \mathrm{a}$ & $31,2 \pm 3,7 \mathrm{ab}$ & $44,69 \pm 6,8 \mathrm{ab}$ \\
\hline
\end{tabular}

Media \pm SD (Desviación estándar). Promedios con letras diferentes en una misma columna presentan diferencias significativas (Tukey, $\mathrm{p}>0.05$ ).

primeros componentes principales son un grupo de combinaciones lineales, calculados para describir la varianza de la muestra y explican un total del $75,37 \%$ de la varianza de los datos experimentales; esto indica, que las dos combinaciones lineales principales describen un porcentaje mayoritario entre todas las muestras.

La deshidratación por aire caliente influye en cambios sensoriales de las muestras, muy marcados en la textura, que se pueden ver afectados por diferentes variables del proceso, como la temperatura, la velocidad del aire o el tiempo de exposición (Moreno-Guarin et al. 2012). Se observa una clara diferencia entre las muestras de tomate deshidratado, indicando una diferencia significativa en las propiedades texturales de las muestras estudiadas, formando tres grupos con características similares: uno, por las muestras 2,3 y 4; otro, por las muestras 1,5 y 6 y la muestra número 7 . Las muestras 1,5 y 6 , se caracterizan por tener una dureza y la masticabilidad más elevada; este parámetro, se define como la energía requerida para masticar un alimento sólido antes de tragarlo (Szczesniak, 2002); se puede correlacionar con la dureza, pues al ser un alimento más duro, se va a requerir más energía para masticarlo. Las muestras 2, 3 y 4 


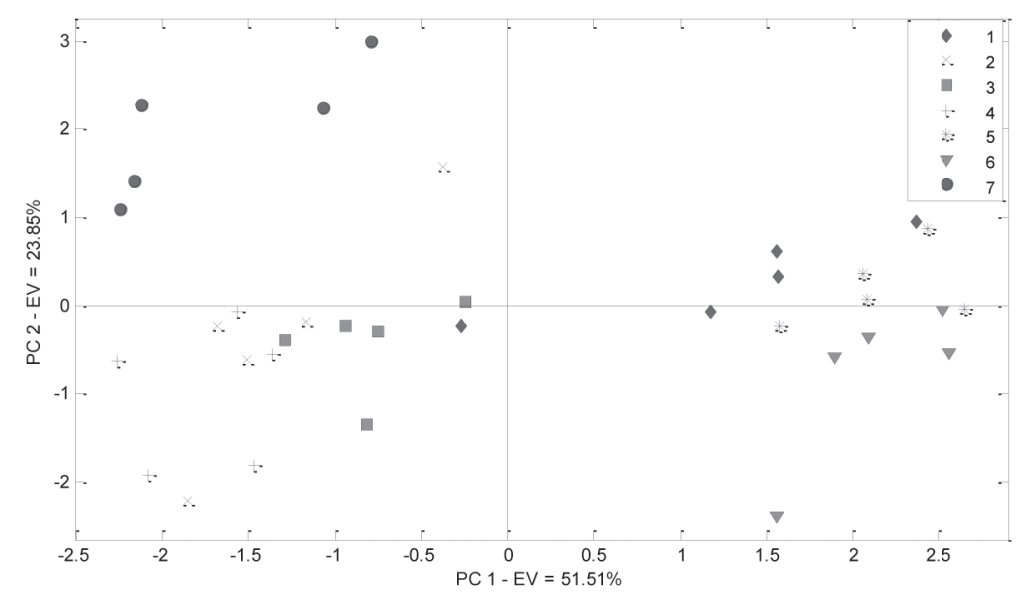

Figura 1: Análisis de las muestras de tomate deshidratado sobre el plano definido por las dos primeras componentes principales.

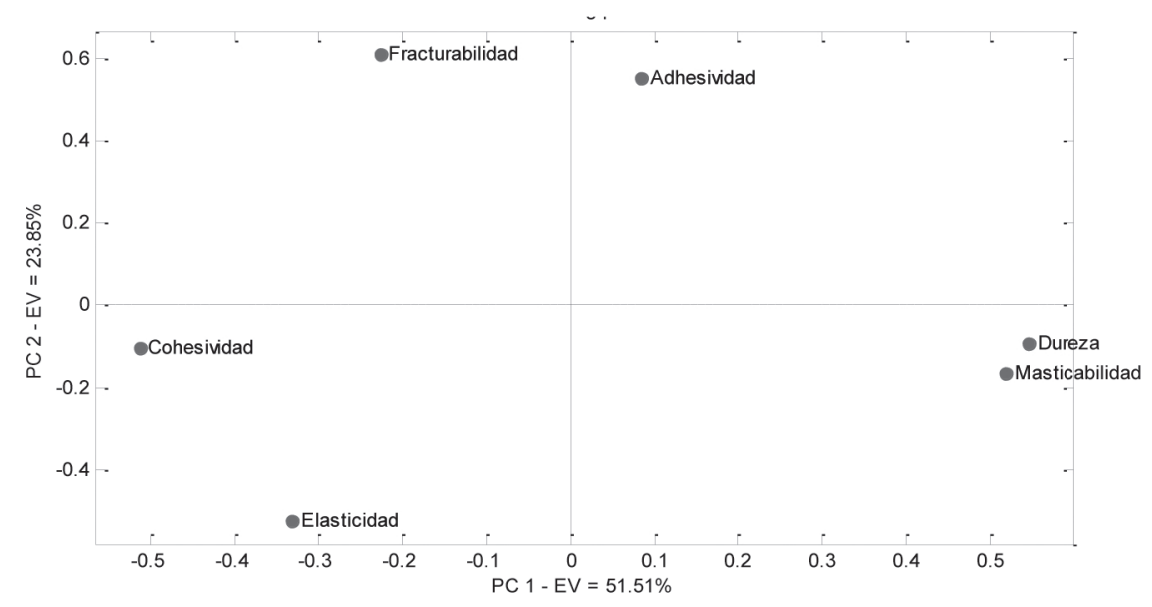

Figura 2: Análisis de las propiedades texturales en muestras de tomate deshidratado sobre el plano definido por las dos primeras componentes principales.

muestran valores más altos de cohesividad y de elasticidad, que en el espacio PCA, antagonistas de la masticabilidad, lo cual, indica que estas muestras son más suaves y, por lo tanto, más fáciles de consumir. La fracturabilidad es asociada por los consumidores a la crocancia, lo que puede llevar a un aumento de su apetencia para ser consumido directamente como snack; la única muestra que presenta un valor de fracturabilidad significativo con respecto a las otras muestras es la 7 .

El análisis microbiológico refleja que los valores referenciados en las diferentes normas se cumplen para coliformes totales y Salmonella (Gilbert et al. 2000; Ministerio de Salud de Perú, 2003; Ministerio da Saude Brasil, 1978); sin embargo, para el caso de los mohos y las levaduras, sólo las muestras 1, 3, 4 y 7 cumplen con la referencia establecida por la norma peruana (Ministerio de Salud de Perú, 2003) y la muestra 2 sobrepasa el máximo de mesófilos aerobios, determinado por la norma británica (Gilbert et al. 2000) (Tabla 3). Estos resultados, se pueden relacionar con mala manipulación, ya que los mesófilos regularmente son microorganismos del ambiente y propios de la flora, que se encuentra en la piel de los humanos y ambientes (Pérez et al. 1998). Adicionalmente, los parámetros fisicoquímicos de actividad de agua y humedad que, al ser más altos de lo que convencionalmente se encuentra en un producto deshidratado, promueven el crecimiento bacteriano, afectando así la calidad del producto. 
Tabla 3. Calidad microbiológica para muestras de tomate deshidratado comercial.

\begin{tabular}{|c|c|c|l|c|}
\hline Muestra & $\begin{array}{c}\text { Coliformes } \\
\text { totales } \\
\text { UFC/g }\end{array}$ & $\begin{array}{c}\text { Recuento de } \\
\text { Mesófilos } \\
\text { UFC/g }\end{array}$ & $\begin{array}{c}\text { SalmonellaPresencia/ } \\
\text { Ausencia }\end{array}$ & $\begin{array}{c}\text { Mohos y levaduras } \\
\text { UFC/g }\end{array}$ \\
\hline 1 & $<3$ & 99000 & Negativa & 70 \\
\hline 2 & $<3$ & 220000 & Negativa & 18000 \\
\hline 3 & $<3$ & 4000 & Negativa & 30 \\
\hline 4 & $<3$ & $<10$ & Negativa & 200 \\
\hline 5 & $<3$ & 36000 & Negativa & 1100 \\
\hline 6 & $<3$ & 2000 & Negativa & $<10$ \\
\hline 7 & $<3$ & 6100 & Negativa & $<10$ \\
\hline
\end{tabular}

Estudios similares se presentan en la evaluación microbiológica de muestras de tomate deshidratado comercial, con recuentos de coliformes, mesófilos aerobios, hongos y levaduras menores a 10UFC/g (Monsalve \& Machado, 2007; Sohail et al. 2011); estos resultados, se favorecen con el contenido de agua presente en el producto. Otros estudios encuentran microorganismos, como mesófilos aerobios (56000UFC/g) y hongos (3600UFC/g), donde influyen las condiciones de almacenamiento y la exposición al ambiente; algunas muestras ni cumplen con los parámetros mínimos de calidad (Idah \& Aderibigbe, 2007; Sohail et al. 2011).

Análisis sensorial: El análisis sensorial con consumidores indica que la muestra 7 es la preferida por los consumidores; seguida de la muestra 1 y la 4 . Correlacionando estos resultados con las medidas instrumentales, se observa que la muestra 7 está claramente diferenciada, en cuanto a propiedades texturales; la variable más influyente es la fracturabilidad, indicando que es una muestra más fracturable y con atributos, como la crocancia. El parámetro de textura para la aceptación del consumidor es la fracturabilidad de la muestra; también los panelistas afirmaron que el color más cercano al del tomate fresco es el aceptado para su consumo.

En general, se observa una gran heterogeneidad entre las muestras de tomate deshidratado encontradas en el comercio local, por lo cual, se hace necesario impulsar la reglamentación de parámetros de calidad fisicoquímica y microbiológica del producto. Las pruebas fisicoquímicas con diferencias significativas entre las muestras son el color y la textura, parámetros primordiales para el estudio de muestras deshidratadas; el color debe ser muy cercano al del tomate fresco y las muestras deben tener propiedades texturales, como la fracturabilidad, para asegurar aceptación sensorial. Adicionalmente, es importante evaluar humedad y actividad de agua en estudios experimentales de tomate deshidratado, con el fin de evitar reacciones de deterioro.
Conflicto de intereses: El manuscrito fue preparado y revisado con la participación de todos los autores, quienes declaramos que no existe ningún conflicto de intereses que ponga en riesgo la validez de los resultados presentados. Financiación: Este estudio fue financiado por el programa Semilleros de creación e investigación de la División de Investigación de la sede Bogotá de la Universidad Nacional de Colombia (4to corte) y el proyecto "Estrategias para selección de indicadores tecnológicos en procesos de deshidratación de frutas y hortalizas utilizando herramientas de automatización y control", financiado por Colciencias.

\section{BIBLIOGRAFÍA}

1. AKDENIZ, B.; KAVAK, D.D.; BAĞDATLIOĞLU, N. 2012. Use of factorial experimental design for analyzing the effect of storage conditions on color quality of sundried tomatoes. Sci. Res. Essays. 7(4):477-489.

2. AOAC. 2002. Official Methods of Analysis. Association of Analytical Chemists. 920.151. total solids in fruit and fruit products.

3. ASHEBIR, D.; JEZIK, K.; WEINGARTEMANN, H.; GRETZMACHER, R. 2009. Change in color and other fruit quality characteristics of tomato cultivars after hot-air drying at low final-moisture content. Int. J. Food Sci. Nutr. 60(7):308-315.

4. BARREIRO, J.A.; MILANO, M.; SANDOVAL, A.J. 1997. Kinetics of colour change of double concentrated tomato paste during thermal treatment. J. Food Eng. 33(3-4):359-371.

5. BATU, A. 2004. Determination of acceptable firmness and colour values of tomatoes. J. Food Eng. 61(3):471-475. 
6. BROOKS, M.S.; GHALY, A.E.; ABOU EL-HANA, N.H. 2008. Effect of osmotic pre-treatment on the airdrying behavior and quality of plum tomato pieces. Int. J. Food Eng. 4(5):1-24.

7. CHANG, C.-H.; LIN, H.-Y.; CHANG, C.-Y.; LIU, Y.-C. 2006. Comparisons on the antioxidant properties of fresh, freeze-dried and hot-air-dried tomatoes. J. Food Eng. 77(3):478-485.

8. GBX. 2008. Fast-lab water activity meter. Disponible desde Internet en: http://www.gbxonline.com/Eng_ AW.html (con acceso 04/11/2012).

9. HEREDIA, A.; BARRERA, C.; ANDRÉS, A. 2007. Drying of cherry tomato by a combination of different dehydration techniques. Comparison of kinetics and other related properties. J. Food Eng. 80(1):111-118.

10. HOSSAIN, M.A.; AMER, B.M.A.; GOTTSCHALK, K. 2008. Hybrid solar dryer for quality dried tomato. Drying Techn. 26(12):1591-1601.

11. ICONTEC. 1999. NTC 4592. Productos de frutas y verduras. determinación del $\mathrm{pH}$. Instituto colombiano de normas tecnicas. Tecnicas (Ed.). Bogotá.

12. IDAH, P.A.; ADERIBIGBE, B.A. 2007. Quality changes in dried tomatoes stored in sealed polythene and open storage Systems. Leonardo Electr. J. Practices Techn. 6(10):1-6.

13. INVIMA. 1998. Manual de técnicas de análisis para el control de calidad microbiológico de alimentos para consumo humano. Bogotá (Colombia).

14. KEK, S.P.; CHIN, N.L.; YUSOF, Y.A. 2013. Direct and indirect power ultrasound assisted pre-osmotic treatments in convective drying of guava slices. Food Biopr. Proces. 91(4):495-506.

15. KHAZAEI, J.; CHEGINI, G.-R.; BAKHSHIANI, M. 2008. A novel alternative method for modeling the effects of air temperature and slice thickness on quality and drying kinetics of tomato slices: superposition technique. Drying Techn. 26(6):759-775.

16. GILBERT, R.J.; DE LOUVOIS, J.; DONOVAN, T.; LITTLE, C.; NYE, K.; RIBEIRO, C.D.; RICHARDS, J.; ROBERTS, D.; BOLTON, F.J. (PHLS Advisory Committee for Food and Dairy Products). 2000. Guidelines for the microbiological quality of some ready-to-eat foods sampled at the point of sale. Commun. Dis. Public Health. 3:163-167.
17. LOZANO, J.E.; IBARZ, A. 1997. Colour changes in concentrated fruit pulp during heating at high temperatures. J. Food Eng. 31(3):365-373.

18. MINISTÉRIO DA SAÚDE BRASIL. 1978. Resolução CNNPA n ${ }^{\circ} 12$, de 1978 Frutas secas ou dessecadas (Vol. 12-1978, p.75). Brasil: Agência Nacional de Vigilância Sanitária.

19. MINISTERIO DE LA PROTECCIÓN SOCIAL DE COLOMBIA. 2011. Proyecto de resolución Frutas. Bogotá. p.2.

20. MINISTERIO DE SALUD DEL PERÚ. 2003. Norma sanitaria que establece los criterios microbiológicos de calidad sanitaria e inocuidad para los alimentos y bebidas de consumo humano De los grupos de alimentos y criterios microbiológicos (Vol. 615-2003, p.24).

21. MONSALVE, J.; MACHADO, M. 2007. Evaluación de dos metodos de deshidratación del tomate (Lycopersicom esculentum Mill) variedad manzano. Multiciencias. 7(3):256-265.

22. MONTGOMERY, D. 2002. Diseño y analisis de experimentos (2a ed.). Mexico D.F. Wiley Editors. 686p.

23. MORENO-GUARIN, D.; SIERRA-HOYOS, H.; DIAZ-MORENO, C. 2012. Color y textura, caracteristicas asociadas a la calidad del tomate deshidratado. Vitae. 19:201-203.

24. OLORUNDA, A.O.; AWORH, O.C.; ONUOHA, C.N. 1990. Upgrading quality of dried tomato: effects of drying methods, conditions and pre-drying treatments. J. Sci. Food Agr. 52(4):447-454.

25. PÉREZ, M.; BELMONTE, S.; MARTÍNEZ, J. 1998. Estudio microbiológico de los alimentos elaborados en comedores colectivos de alto riesgo. Rev. Esp. Salud Publica. 72(1):67-75.

26. POLENTA, G.; LUCANGELI, C.; BUDDE, C.; GONZÁLEZ, C.B.; MURRAY, R. 2006. Heat and anaerobic treatments affected physiological and biochemical parameters in tomato fruits. LWT - Food Sci. Techn. 39(1):27-34.

27. RATTI, C. 2001. Hot air and freeze-drying of high-value foods: a review. J. Food Eng. 49(4):311-319.

28. RATTI, C. (Ed.). 2009. Advances in food Dehydration (1a ed.): Taylor and Francis group. 477p. 
29. SHI, J.; MAGUER, M.L.; KAKUDA, Y.; LIPTAY, A.; NIEKAMP, F. 1999. Lycopene degradation and isomerization in tomato dehydration. Food Res. Int. 32(1):1521.

30. SHIN, S.; BHOWMIK, S.R. 1995. Thermal kinetics of color changes in pea puree. J. Food Eng. 24(1):77-86.

31. SIDEL, J.L.; MOSKOWITZ, H.R. 1971. Magnitude and hedonic scales of food acceptability. J. Food Sci. 36(4):677-680.

32. SIDEL, J.L.; STONE, H. 1976. Experimental design and analysis of sensory tests. Food Techn. 30(11):32-35.

33. SOHAIL, M.; AYUB, M.; AHMAD, I.; ALI, B.; DAD, F. 2011. Physicochemical and microbiological evaluation of sun dried tomatoes in comparison with fresh tomatoes. Pakistan J. Biochem. Molec. Biol. 44(3):106-109.

34. SZCZESNIAK, A.S. 2002. Texture is a sensory property. Food Qual. Pref. 13(4):215-225.

35. THYBO, A.K.; EDELENBOS, M.; CHRISTENSEN, L.P., SØRENSEN, J.N.; THORUP-KRISTENSEN, K. 2006. Effect of organic growing systems on sensory quality and chemical composition of tomatoes. LWT - Food Sci. Techn. 39(8):835-843.

36. ZANONI, B.; PERI, C.; NANI, R.; LAVELLI, V. 1998. Oxidative heat damage of tomato halves as affected by drying. Food Res. Int. 31(5):395-401.

Recibido: Mayo 30 de 2013

Aceptado: Marzo 17 de 2014

Como citar.

Moreno, D.C.; Sierra, H.M.; Díaz-Moreno, C. 2014. Evaluación de parámetros de calidad fisicoquímica, microbiológica y sensorial en tomate deshidratado comercial (Lycopersicum esculentum). Rev. U.D.C.A Act. \& Div. Cient. 17(1): 131-138. 